\title{
REFINABLE FUNCTIONS WITH NON-INTEGER DILATIONS
}

\author{
XIN-RONG DAI, DE-JUN FENG, AND YANG WANG
}

\begin{abstract}
Refinable functions and distributions with integer dilations have been studied extensively since the pioneer work of Daubechies on wavelets. However, very little is known about refinable functions and distributions with non-integer dilations, particularly concerning its regularity.

In this paper we study the decay of the Fourier transform of refinable functions and distributions. We prove that uniform decay can be achieved for any dilation. This leads to the existence of refinable functions that can be made arbitrarily smooth for any given dilation factor. We exploit the connection between algebraic properties of dilation factors and the regularity of refinable functions and distributions. Our work can be viewed as a continuation of the work of Erdös [6], Kahane [11] and Solomyak [19] on Bernoulli convolutions. We also construct explicitly a class of refinable functions whose dilation factors are certain algebraic numbers, and whose Fourier transforms have uniform decay. This extends a classical result of Garsia [9].
\end{abstract}

\section{INTRODUCTION}

In this paper we study the refinement equation

$$
f(x)=\sum_{j=0}^{m} c_{j} f\left(\lambda x-d_{j}\right), \quad \sum_{j=0}^{m} c_{j}=|\lambda|
$$

where $\lambda \in \mathbb{R}$ with $|\lambda|>1$ and all $c_{j}, d_{j}$ are real. It is well known that up to a scalar multiple the above refinement equation has a unique distribution solution $f$, which is furthermore compactly supported. We shall refer to the distribution solution $f(x)$ of $(1.1)$ with $\hat{f}(0)=1$ the solution to (1.1). For the refinement equation (1.1), the value $\lambda$ is called the dilation factor of the refinement equation, and $\left\{d_{j}\right\}$ the translation set or simply the translations. The coefficients $\left\{c_{j}\right\}$ are called the weights (even though they can be negative). For simplicity we shall call a solution $f(x)$ to (1.1) a $\lambda$-refinable distribution (function) with translations $\left\{d_{j}\right\}$.

The first author is supported in part by Department of Education of Zhejiang Province (Grant 20051495). The second author is supported in part by the direct grant and RGC grant in CUHK, Fok Ying Tong Education Foundation and NSFC (10571100).

The third author is supported in part by the National Science Foundation, grants DMS-0070586 and DMS-0139261. 
The questions we study concern the regularity of $\lambda$-refinable functions or distributions. Particularly we are interested in refinable functions whose dilation factors are non-integers. For example, is it possible to find a 3/2-refinable function that is smooth? More generally, is it possible to find a smooth $\lambda$-refinable function for any $\lambda$ with $|\lambda|>1$ ?

Refinable functions play a fundamental role in the construction of compactly supported wavelets and in the study of subdivisions schemes in approximation theory, see Daubechies [4] and Cavaretta, Dahman and Micchelli [1]. In both cases the dilation factors are restricted to integers, as are the translations. It is well known that for any integer dilation $\lambda$ there exist $\lambda$-refinable functions with integer translations that can be made arbitrarily smooth. The simplest example is the $B$-spline $B_{m}(x)$, which is obtained by convolving $\chi_{[0,1)}$ with itself $m$ times. $B_{m}$ is $\lambda$-refinable for any integer $\lambda,|\lambda|>1$. The $B$-splines have important applications in subdivision schemes and computer aided geometric designs. With integer dilations and translations one may impose strong constraints on the weights while still attaining smoothness. The most important example is the construction of a class of smooth refinable functions whose integer translates are mutually orthogonal that began with the seminal work of Daubechies [3] leading to compactly supported orthonormal wavelets.

But the regularity question becomes more complicated, and perhaps more interesting from the pure analysis point of view, when the dilation factors $\lambda$ are non-integers, particularly when the translations are still restricted to integers. There is a strong connection with number theory that still needs to be fully exploited. The regularity of refinable functions and distributions seem to be strongly affected by algebraic properties of the dilation factors.

One way to characterize regularity is to consider the decay of $\widehat{f}$. Let $f(x)$ be a distribution. We say $\widehat{f}$ has uniform decay at infinity if $\widehat{f}(\xi)=O\left(|\xi|^{-\gamma}\right)$ for some $\gamma>0$. Suppose that $\widehat{f}$ has uniform $\gamma$-decay at infinity. Let $f^{* n}(x):=f * f * \cdots * f(x)$ in which $f$ convolves with itself $n$ times. Then $\widehat{f^{* n}}=\widehat{f}^{n}$, which has uniform $n \gamma$-decay at infinity. By taking $n$ large one can make $f^{* n}$ an arbitrarily smooth function. Furthermore, if $f$ is $\lambda$-refinable then so is $f^{* n}$. In fact if $f$ is $\lambda$-refinable with integer translations then so is $f^{* n}$. Thus we shall focus on the following question: Given any $\lambda \in \mathbb{R}$ with $|\lambda|>1$, is there a $\lambda$-refinable distribution $f(x)$ such that $\widehat{f}$ has uniform decay at infinity? What if the translations are required to be integers? 
A large amount of literature have been devoted to this questions in the case of Bernoulli convolutions, which are the solutions to

$$
f(x)=\frac{|\lambda|}{2} f(\lambda x)+\frac{|\lambda|}{2} f(\lambda x-1)
$$

Many of these studies apply to the more general setting of integer translations as well. Erdös [5] proved that under the integer translations setting any $\lambda$-refinable distribution $f$ has $\widehat{f}(\xi) \not 0$ as $|\xi| \rightarrow \infty$ as long as $\lambda$ is a Pisot number, i.e. an algebraic integer whose algebraic conjugates are all inside the unit circle. This immediately implies that $f$ cannot be in $L^{1}$. It remains an open question whether Pisot numbers are the only dilations for which one cannot construct $L^{1}$ refinable functions with integer translations. Also under the integer translation setting Kahane [11] proved that $\widehat{f}$ does not have uniform decay at infinity for any $\lambda$-refinable distribution if $\lambda$ is a Salem number, i.e. an algebraic integer whose algebraic conjugates are all inside or on the unit circle, assuming that some conjugates lie actually on the unit circle. (Both Erdös and Kahane established their results for Bernoulli convolutions, but with some technical twisting we may extend their results to the more general setting, see Appendix.) In the positive direction, Garsia [9] proved that the Bernoulli convolution $f(x)$ of $(1.2)$ is in $L^{\infty}$, if the dilation $\lambda$ is an algebraic integer whose algebraic conjugates are all outside the unit circle and the constant term for its minimal polynomial is \pm 2 . Garsia's result remains today as the only explicitly known class of Bernoulli convolutions that are in $L^{1}$. Feng and Wang [7] explicitly constructed a large class of algebraic integer dilations $\lambda$ for which the corresponding Bernoulli convolutions are not in $L^{2}$. In the generic setting Solomyak [19] proved that for almost all dilations $\lambda \in(1,2)$ the corresponding Bernoulli convolution is in $L^{1}$, and more recently, Peres and Schlag [16] proved that the Fourier transform of the Bernoulli convolution has uniform decay at infinity for almost all dilations $\lambda \in(1,2)$. It is not clear whether the latter result holds for almost all $\lambda \in(1, \infty)$. Our results in this paper can be viewed as an extension of the aforementioned studies.

When the dilations and translations are both integers and the weights are nonnegative, the uniform decay property can be characterized completely:

Theorem 1.1. Let $f(x)$ be the distribution solution to the refinement equation

$$
f(x)=\sum_{j=0}^{m} c_{j} f\left(\lambda x-d_{j}\right), \quad \sum_{j=0}^{m} c_{j}=|\lambda|
$$

where $\lambda \in \mathbb{Z}$ and $c_{j}>0, d_{j} \in \mathbb{Z}$ for all $j$. Then the following are equivalent: 

(A) $\widehat{f}$ has uniform decay at $\infty$.
(B) $f \in L^{\infty}(\mathbb{R})$.
(C) $f \in L^{1}(\mathbb{R})$.
(D) $\lim _{|\xi| \rightarrow \infty} \widehat{f}(\xi)=0$.
(E) $\widehat{f}(n)=0$ for all $n \in \mathbb{Z} \backslash\{0\}$.
(F) For any $n \in \mathbb{Z} \backslash\{0\}$ there exists a $k=k(n)>0$ such that $P\left(\lambda^{-k} n\right)=0$, where $P(\xi):=\frac{1}{|\lambda|} \sum_{j=0}^{m} c_{j} e\left(-d_{j} \xi\right)$ with $e(t):=e^{2 \pi i t}$.

The trigonometric polynomial $P(\xi)$ is called the symbol of the refinement equation. Note that Theorem 1.1 can be partially extended to the case of rational dilations.

Theorem 1.2. Let $f(x)$ be the distribution solution to the refinement equation

$$
f(x)=\sum_{j=0}^{m} c_{j} f\left(\lambda x-d_{j}\right), \quad \sum_{j=0}^{m} c_{j}=|\lambda|
$$

where $\lambda \in \mathbb{Q},|\lambda|>1$ and $c_{j}>0, d_{j} \in \mathbb{Z}$ for all $j$. Suppose that for any $n \in \mathbb{Z} \backslash\{0\}$ there exists a $k=k(n)>0$ such that $P\left(\lambda^{-k} n\right)=0$, where $P(\xi)$ is the symbol of the refinement equation. Then the following hold:
(A) $\widehat{f}$ has uniform decay at $\infty$.
(B) $f \in L^{\infty}(\mathbb{R})$.
(C) $\widehat{f}(n)=0$ for all $n \in \mathbb{Z} \backslash\{0\}$.

We remark that both Theorem 1.1 and Thereom 1.2 hold under the weaker assumption that $|P(\xi)|<1$ for all $\xi \in \mathbb{R} \backslash \mathbb{Z}$. This is rather easy to see from the proof. Also, Theorem 1.2 holds for any dilation $\lambda$, but unless $\lambda^{k} \in \mathbb{Q}$ for some $k>0$ there exists no refinement equations satisfying the hypotheses of the theorem.

One of the well known questions concerning refinable functions is whether one can construct arbitrarily smooth refinable functions for rational dilations such as $\lambda=3 / 2$. Here we answer this question.

Corollary 1.3. Let $\lambda=p / q$ where $p>|q|$ are integers and $(p, q)=1$. Then the refinable distribution satisfying

$$
f(x)=\frac{1}{|q|} \sum_{j=0}^{p-1} f\left(\frac{p}{q} x-j\right)
$$


is in $L^{\infty}(\mathbb{R})$, and $\widehat{f}$ has uniform decay at $\infty$. As a consequence, for any $k \geq 0$ there exists a compactly supported $\lambda$-refinable function $f$ with integer translations and nonnegative weights such that $f$ is in $C^{k}$.

In Section 2 we shall explicitly construct a differentiable 3/2-refinable function.

Theorem 1.4. Let $\lambda \in \mathbb{R}$ with $|\lambda|>1$ and $c_{j}>0$ for all $j$. Let $f=f_{t}$ be the distribution solution of the refinement equation

$$
f(x)=\sum_{j=0}^{m} c_{j} f\left(\lambda x-d_{j}\right), \quad \sum_{j=0}^{m} c_{j}=|\lambda|,
$$

where $d_{0}, d_{1}$ are fixed and distinct, and $d_{m}=t$ with $t$ being a paramenter. Then there exists a $E:=E_{\lambda} \subset \mathbb{R}$ independent of $\left\{c_{j}\right\}_{j=0}^{m}$ with $\operatorname{dim}_{H}(E)=0$, such that $\hat{f}_{t}$ has uniform decay at infinity for each $t \in \mathbb{R} \backslash E$.

Here $\operatorname{dim}_{H}$ denotes the Hausdorff dimension (see e.g., [8] for a definition). Obviously the set $E$ has Lebesgue measure 0 . By taking convolution of $f_{t}(x)$ with itself repeatedly we easily obtain:

Corollary 1.5. Let $\lambda \in \mathbb{R}$ with $|\lambda|>1$. Then for any $k \geq 0$ there exists a compactly supported $\lambda$-refinable function with nonnegative weights that is in $C^{k}$.

Note that in the language of self-similar measures the above corollary states that for any $\lambda$ with $|\lambda|>1$ there exists a self-similar measure with contraction $\lambda^{-1}$ whose density can be arbitrarily smooth. Our next theorem is an extension of Garsia [9].

Theorem 1.6. Let $p(x)=\sum_{j=0}^{n} a_{j} x^{j} \in \mathbb{Z}[x]$ be irreducible (but not necessarily monic) such that all roots of $p(x)$ are outside the unit circle. Let $\lambda$ be a real root of $p(x)$ and $f(x)$ be the distribution solution to

$$
f(x)=\frac{|\lambda|}{\left|a_{0}\right|} \sum_{j=0}^{\left|a_{0}\right|-1} f(\lambda x-j) .
$$

Then $\widehat{f}$ has uniform decay at infinity. Furthermore, $f \in L^{\infty}$.

Garsia [9] proved that $f$ is $L^{\infty}$ in the case $a_{n}=1$ and $a_{0}= \pm 2$. No uniform decay property was established in [9], however. 


\section{Proof of Theorem 1.1 And Theorem 1.2}

We consider in this section refinable distributions with integer translations and integer or rational dilations. First we introduce a notation. For any $x \in \mathbb{R}$ we let $\|x\|_{\mathbb{Z}}$ denote the distance of $x$ to the integers $\mathbb{Z}$. Thus $\|x\|_{\mathbb{Z}} \leq 1 / 2$. We may without loss of generality consider the refinement equation

$$
f(x)=\sum_{j=0}^{m} c_{j} f\left(\lambda x-d_{j}\right), \quad \sum_{j=0}^{m} c_{j}=|\lambda|
$$

where $\lambda \in \mathbb{Z}$ or $\lambda \in \mathbb{Q}, c_{j}>0$ for all $j$ and $0=d_{0}<d_{1}<\cdots<d_{m}$ are in $\mathbb{Z}$. Furthermore, we assume that $\operatorname{gcd}\left(d_{1}, d_{2}, \ldots, d_{m}\right)=1$.

Lemma 2.1. Let $\lambda=p / q \in \mathbb{Q}$ with $p>|q|$ and $(p, q)=1$. Let $Q(t)$ be a trigonometric polynomial with $Q(0)=1$ and $|Q(t)|<1$ for any $t \notin \mathbb{Z}$. Furthermore, $Q(t)$ has the property that for any $n \in \mathbb{Z} \backslash\{0\}$ there exists a $k>0$ such that $Q\left(\lambda^{-k} n\right)=0$. Fix an $\varepsilon>0$. Suppose that $|t|>\varepsilon$ and $\|t\|_{\mathbb{Z}}<\varepsilon$. Then there exists an $\ell \in \mathbb{N}$ such that $|g(t)| \leq C \varepsilon|\lambda|^{-\ell}\left|g\left(\lambda^{-\ell} t\right)\right|$, where $g(t):=\prod_{j=1}^{\infty} Q\left(\lambda^{-j} t\right)$ and $C=\max \left|Q^{\prime}(t)\right|$. In particular, $g$ has uniform decay at infinity.

Proof. Write $t=n+\delta$ where $n \in \mathbb{Z}$ and $|\delta|=\|t\|_{\mathbb{Z}}$. Note that $|t|>\varepsilon$, so $n \neq 0$. Therefore there exists an $\ell>0$ such that $Q\left(\lambda^{-\ell} n\right)=0$. This means that

$$
\left|Q\left(\lambda^{-\ell} t\right)\right|=\left|Q\left(\lambda^{-\ell} t\right)-Q\left(\lambda^{-\ell} n\right)\right| \leq C\left|\lambda^{-\ell} \delta\right|<C \varepsilon|\lambda|^{-\ell} .
$$

Therefore

$$
|g(t)|=\prod_{j=1}^{\infty}\left|Q\left(\lambda^{-j} t\right)\right| \leq Q\left(\lambda^{-\ell} t\right) \prod_{j=\ell}^{\infty}\left|Q\left(\lambda^{-j} t\right)\right| \leq C \varepsilon|\lambda|^{-\ell}\left|g\left(\lambda^{-\ell} t\right)\right| .
$$

The decay property of $g$ can now be established. Let $M=\sup \left\{|Q(t)|:\|t\|_{\mathbb{Z}} \geq \varepsilon\right\}<1$. For any $t \in \mathbb{R}$ with $|t|>|\lambda|$, we can uniquely write $t=\lambda^{N} s$ for some $s$ with $|s| \in[1,|\lambda|]$ and $N \in \mathbb{N}$. Now $g$ is an analytic function, and so it is bounded on $[-|\lambda|,|\lambda|]$, say by the constant $K>0$. Suppose that $\left\|\lambda^{-1} t\right\|_{\mathbb{Z}} \geq \varepsilon$. Then $\left|Q\left(\lambda^{-1} t\right)\right| \leq M$. Hence $|g(t)|=$ $\left|g\left(\lambda^{N} s\right)\right| \leq M\left|g\left(\lambda^{N-1} s\right)\right|$. On the other hand, suppose that $\left\|\lambda^{-1} t\right\|_{\mathbb{Z}}<\varepsilon$. Then

$$
|g(t)| \leq\left|g\left(\lambda^{-1} t\right)\right| \leq C \varepsilon|\lambda|^{-\ell}\left|g\left(\lambda^{N-\ell-1} s\right)\right| .
$$

Take $\rho \in(M, 1)$ such that $\rho^{k+1} \geq C \varepsilon|\lambda|^{-k}$ for all $k \in \mathbb{N}$. This $\rho$ clearly exists if we take $\varepsilon$ to be small enough. Then (2.2) becomes $|g(t)| \leq \rho^{\ell+1}\left|g\left(\lambda^{N-\ell-1} s\right)\right|$. Combining with the 
case $\left\|\lambda^{-1} t\right\|_{\mathbb{Z}} \geq \varepsilon$ we now have $|g(t)|=\left|g\left(\lambda^{N} s\right)\right| \leq \rho^{N} K$. The uniform decay property is now established by taking $\gamma=\log \rho^{-1} / \log |\lambda|$, and $\left|g\left(\lambda^{N} s\right)\right| \leq K|\lambda|^{-\gamma N}$.

Proof of Theorem 1.1. It is clear that $(\mathrm{B}) \Rightarrow(\mathrm{C}) \Rightarrow(\mathrm{D})$.

$(D) \Rightarrow(E)$. We have $\widehat{f}(\xi)=\widehat{f}(0) \prod_{j=1}^{\infty} P\left(\lambda^{-j} \xi\right)$. In particular $\widehat{f}\left(\lambda^{k} \xi\right)=\widehat{f}(\xi) \prod_{j=0}^{k-1} P\left(\lambda^{j} \xi\right)$. This implies that $\widehat{f}\left(\lambda^{k} n\right)=\widehat{f}(n)$. By letting $k \rightarrow \infty$ we have $\widehat{f}(n)=0$ for all $n \in \mathbb{Z} \backslash\{0\}$.

$(E) \Rightarrow(F)$. Again we invoke the property $\widehat{f}(\xi)=\widehat{f}\left(\lambda^{-k} \xi\right) \prod_{j=1}^{k} P\left(\lambda^{-j} \xi\right)$. Since $\widehat{f}(0) \neq 0$ and $\widehat{f}(\xi)$ is analytic, it follows from $\widehat{f}(n)=0$ that $\prod_{j=1}^{k} P\left(\lambda^{-j} n\right)=0$ when $k$ is large enough. (F) now follows.

$(F) \Rightarrow(B)$. By our convention it is assumed that $\widehat{f}(0)=1$. It is known that $f$ is in fact a probability measure, see e.g. Falconer [8]. Now $f$ is compactly supported. Define $F(x)=\sum_{n \in \mathbb{Z}} f(x-n)$. Then $F$ is a Radon measure, and hence a distribution. We have $F=f * \delta_{\mathbb{Z}}$ where $\delta_{\mathbb{Z}}:=\sum_{n \in \mathbb{Z}} \delta(x-n)$. The Poisson Summation Formula yields $\widehat{F}=\widehat{f} \cdot \widehat{\delta_{\mathbb{Z}}}=\widehat{f} \cdot \delta_{\mathbb{Z}}$. Therefore $\widehat{F}=\delta$, and hence $F=1$. This implies that $f \in L^{\infty}$.

$(A) \Rightarrow(D)$. This is clear.

$(F) \Rightarrow(A) . P(\xi)$ satisfies the hypotheses of Lemma 2.1. By the lemma $\widehat{f}(\xi)=\prod_{j=1}^{\infty} P\left(\lambda^{-j} \xi\right)$ has uniform decay at infinity.

Proof of Theorem 1.2. The proof is identical to the proof of Theorem 1.1, and we only give a brief explanation. Clearly, the hypotheses of the theorem implies $(\mathrm{C})$, that is, $\widehat{f}(n)=0$ for all $n \in \mathbb{Z} \backslash\{0\}$. The argument used to prove $(\mathrm{F}) \Rightarrow(\mathrm{B})$ in Theorem 1.1 now applies to prove that $f \in L^{\infty}$. The uniform decay of $\widehat{f}$ is established by Lemma 2.1.

Proof of Corollary 1.3. Let $P(\xi)=\frac{1}{p} \sum_{j=0}^{p-1} e(-j \xi)$ be the symbol of the refinement equation. $P(\xi)$ clearly satisfies the hypotheses of Theorem 1.2. Hence $\widehat{f}$ has uniform decay at infinity, and $f \in L^{\infty}$. Convolving $f$ with itself sufficiently many times yields a $\lambda$-refinable function that can be made arbitrarily smooth.

Example 2.1. We consider the compactly supported refinable function $f(x)$ given by

$$
f(x)=\frac{1}{2} f\left(\frac{3}{2} x\right)+\frac{1}{2} f\left(\frac{3}{2} x+1\right)+\frac{1}{2} f\left(\frac{3}{2} x-1\right) .
$$

$f$ is in $L^{\infty}$ by Corollary 1.3, and $\widehat{f}$ has uniform decay. We prove that $\widehat{f}(\xi)=O\left(|\xi|^{-1}\right)$ as $|\xi| \rightarrow \infty$. This immediately implies that $f^{* n}$ is $(n-2)$-times differentiable. 
To prove this, observe that the symbol of the refinement equation is $P(\xi)=\frac{1+2 \cos (2 \pi \xi)}{3}$. Let $\lambda=3 / 2$ and $\xi=\lambda^{N} s$ where $|s| \in[1, \lambda)$. We prove that $|\widehat{f}(\xi)| \leq K \lambda^{-N}$ for $K=$ $\max _{|s| \leq \lambda}|\widehat{f}(s)|$. A simple check with Maple shows that by taking $\varepsilon=\frac{1.05}{2 \pi}$ we have $M=$ $\max _{\|t\|_{\mathbb{Z} \geq \varepsilon}}|P(t)|<\lambda^{-1}=2 / 3$. Furthermore, another check with Maple shows that $\mid P\left(\frac{n}{3}+\right.$ $t)|\leq C| t \mid$ for some $C$ with $C \varepsilon<\lambda^{-1}=2 / 3$, as long as $n \neq 3 k$ and $|t|<\varepsilon$. As a result, by the estimations used to prove Lemma 2.1, we obtain $\left|f\left(\lambda^{N} s\right)\right| \leq K \lambda^{-N}=K\left(\frac{2}{3}\right)^{N}$.

\section{Proof of Theorem 1.4}

Without loss of generality we may assume that (1.3) is normalized so that $d_{0}=0$ and $d_{1}=1$. Let $P_{t}(\xi)=\frac{1}{|\lambda|} \sum_{j=0}^{m} c_{j} e\left(-d_{j} \xi\right)$. We have

$$
P_{t}(\xi)=\frac{1}{|\lambda|}\left(c_{0}+c_{1} e(-\xi)+c_{m} e(-t \xi)+\sum_{j=2}^{m-1} c_{j} e\left(-d_{j} \xi\right)\right)
$$

and $\widehat{f}_{t}(\xi)=\prod_{j=1}^{\infty} P_{t}\left(\lambda^{-j} \xi\right)$. To prove Theorem 1.4 we establish a series of lemmas.

Lemma 3.1. Let $\lambda>1$. For a fixed $t$ assume that $\widehat{f}_{t}$ has no uniform decay at infinity. Then for any $\varepsilon, \delta>0$ and $N \in \mathbb{N}$, there exist $a \in[1, \lambda)$ and $n>N$ such that both $\left\{0 \leq j \leq n-1:\left\|a \lambda^{j}\right\|_{\mathbb{Z}} \geq \delta\right\}$ and $\left\{0 \leq j \leq n-1:\left\|a t \lambda^{j}\right\|_{\mathbb{Z}} \geq \delta\right\}$ have cardinality less than $\varepsilon n$.

Proof. Assume the lemma is false. Then there exist $\varepsilon, \delta$ and $N$ such that $\{0 \leq j \leq$ $\left.n-1:\left\|a \lambda^{j}\right\|_{\mathbb{Z}} \geq \delta\right\}$ or $\left\{0 \leq j \leq n-1:\left\|a t \lambda^{j}\right\|_{\mathbb{Z}} \geq \delta\right\}$ have cardinality at least $\varepsilon n$ for all $a \in[1, \lambda]$ and $n>N$. Let

$$
M=M_{\delta}=\max \left\{\left|P_{t}(\xi)\right|:\|\xi\|_{\mathbb{Z}} \geq \delta \text { or }\|t \xi\|_{\mathbb{Z}} \geq \delta\right\} .
$$

It is clear $0<M<1$. Let

$$
\mathcal{A}_{n}:=\left\{0 \leq j \leq n-1:\left\|a \lambda^{j}\right\|_{\mathbb{Z}} \geq \delta \text { or }\left\|a t \lambda^{j}\right\|_{\mathbb{Z}} \geq \delta\right\} .
$$

Set $h=\frac{\varepsilon \log (1 / M)}{\log \lambda}$. 
Now any $\xi>0$ can be uniquely written as $\xi=\lambda^{n} s$ for some $s \in[1, \lambda)$ and $n \in \mathbb{Z}$. For $n>N$ we have

$$
\begin{aligned}
\left|\widehat{f}_{t}(\xi)\right| & =\prod_{j=0}^{n-1}\left|P_{t}\left(\lambda^{j} s\right)\right| \cdot\left|\widehat{f}_{t}(s)\right| \\
& \leq C \prod_{j \in \mathcal{A}_{n}}\left|P_{t}\left(\lambda^{j} s\right)\right| \\
& \leq C M^{\varepsilon n} \\
& =C \lambda^{-h n}
\end{aligned}
$$

where $C=\max _{s \in[1, \lambda]}\left|\widehat{f}_{t}(s)\right|$. This shows decay in $\widehat{f}_{t}(\xi)$ for $\xi>0$. However, $\widehat{f}_{t}(-\xi)=\overline{\widehat{f}_{t}(\xi)}$, which also yields decay in $\widehat{f}_{t}(\xi)$ for $\xi<0$. Hence $\widehat{f}_{t}(\xi)$ has uniform decay at $\infty$. This is a contradiction.

Let $\lambda>1$. For any $\delta>0$ and $n \in \mathbb{N}$ define $\mathcal{A}_{\delta, n}(a):=\left\{0 \leq j<n:\left\|\lambda^{j} a\right\|_{\mathbb{Z}} \geq \delta\right\}$. Introduce the sets

$$
\begin{aligned}
E_{\lambda}(n, \varepsilon, \delta) & =\left\{a \in \mathbb{R}:\left|\mathcal{A}_{\delta, n}(a)\right|<\varepsilon n\right\} \text { and } \\
F_{\lambda}(n, \varepsilon, \delta) & =\left\{x / y: x, y \in E_{\lambda}(n, \varepsilon, \delta), 1 \leq y<\lambda\right\} .
\end{aligned}
$$

Lemma 3.2. For any $\varepsilon, \delta>0$ and $\ell \in \mathbb{N}$, if $\widehat{f_{t}}$ does not have uniform decay at infinity then $t \in \bigcap_{k=1}^{\infty} \bigcup_{n=k}^{\infty} F_{\lambda^{\ell}}(n, \varepsilon, \delta)$.

Proof. Assume that $\widehat{f}_{t}$ has no uniform decay at infinity. Set $\varepsilon^{\prime}=\varepsilon /(2 \ell)$. Then by Lemma 3.1, for any $N \in \mathbb{N}$ there exist $a \in[1, \lambda]$ and $n>N$ such that $\left|\mathcal{A}_{\delta, n}(a)\right|<\varepsilon^{\prime} n$ and $\left|\mathcal{A}_{\delta, n}(a t)\right|<\varepsilon^{\prime} n$. Define $m=[n / \ell]$, where $[x]$ denotes the integral part of $x$. Observe that

$$
\left\{0 \leq j<m:\left\|a \lambda^{\ell j}\right\|_{\mathbb{Z}} \geq \delta\right\} \subset\left\{0 \leq j<n:\left\|a \lambda^{j}\right\|_{\mathbb{Z}} \geq \delta\right\}=\mathcal{A}_{\delta, n}(a) .
$$

It follows that the cardinality of $\left\{0 \leq j<m:\left\|a \lambda^{\ell j}\right\|_{\mathbb{Z}} \geq \delta\right\}$ is not exceeding $\varepsilon^{\prime} n \leq \varepsilon m$. Thus $a \in E_{\lambda^{\ell}}(m, \epsilon, \delta)$. An identical argument shows at $\in E_{\lambda^{\ell}}(m, \epsilon, \delta)$. Since $a \in[1, \lambda]$, we have $t \in F_{\lambda^{\ell}}(m, \epsilon, \delta)$. Noting that $m$ can take infintely many integers, we obtain $t \in$ $\bigcap_{k=1}^{\infty} \bigcup_{m=k}^{\infty} F_{\lambda^{\ell}}(m, \epsilon, \delta)$.

Proposition 3.3. Let $\lambda>16$. There exist $\varepsilon_{0}, \delta_{0}>0$ (depending on $\lambda$ ) such that the set $\bigcap_{k=1}^{\infty} \bigcup_{n=k}^{\infty} F_{\lambda}\left(n, \varepsilon_{0}, \delta_{0}\right) \cap[1, \infty)$ has the Hausdorff dimension not exceeding $\log 16 / \log \lambda$.

This proposition is the key ingredient in, and forms the bulk of, the proof of Theorem 1.4. We shall prove Proposition 3.3 later. 
Proof of Theorem 1.4. First we consider the case $\lambda>1$. Pick $\ell \in \mathbb{N}$ so that $\lambda^{\ell}>$ 16. By Proposition 3.3, for any integer $j \geq \ell$, there exist $\varepsilon_{j}, \delta_{j}>0$ such that the set $\bigcap_{k=1}^{\infty} \bigcup_{n=k}^{\infty} F_{\lambda^{j}}\left(n, \varepsilon_{j}, \delta_{j}\right) \cap[1, \infty)$ has the Hausdorff dimension not exceeding $\log 16 /(j \log \lambda)$. Denote

$$
E_{1}=\bigcap_{j \geq \ell}\left(\bigcap_{k=1}^{\infty} \bigcup_{n=k}^{\infty} F_{\lambda^{j}}\left(n, \varepsilon_{j}, \delta_{j}\right) \cap[1, \infty)\right) .
$$

Then $\operatorname{dim}_{H} E_{1}=0$ and it is independent of $\left\{c_{j}\right\}$. For any $t>1$ and $t \notin E_{1}, \widehat{f}_{t}$ has uniform decay at $\infty$ by Lemma 3.2.

We still need to prove that $\widehat{f}_{t}$ has uniform decay at $\infty$ for all $t<1$ except for a set of zero Hausdorff dimension. For $0<t<1$ consider $g(x):=g_{t}(x)=f(t x)$. Then $g$ satisfies the refinement equation

$$
g(x)=\sum_{j=0}^{n} c_{j} g\left(\lambda x-d_{j} t^{-1}\right)
$$

which contains the translations $\left\{0,1, t^{-1}\right\}$. Let $t_{1}=t^{-1}$. Then $\widehat{g_{t}}$ has unform decay at $\infty$ for all $t_{1}>1$ except for a set of zero Hausdorff dimension. Hence there exists a $E_{2} \subset(0,1)$ with $\operatorname{dim}_{H}\left(E_{2}\right)=0$ such that $\widehat{f}_{t}$ has uniform decay for all $t \in(0,1) \backslash E_{2}$. Finally, for $t<0$ we let $h(x):=h_{t}(x)=f\left(-t x+\frac{t}{\lambda-1}\right)$. Then $h=h_{t}$ satisfies

$$
h(x)=\sum_{j=0}^{n} c_{j} h\left(\lambda x+d_{j} t^{-1}-1\right),
$$

which has $\left\{0,1,1-t^{-1}\right\}$ among the translations. Set $t_{2}=1-t^{-1}$, and the same argument now yields the existence of $E_{3} \subset(-\infty, 0)$ with zero Hausdorff dimension such that $\widehat{f}_{t}$ has uniform decay for all $t \in(-\infty, 0) \backslash E_{3}$. The theorem is now proved by letting $E=$ $E_{1} \cup E_{2} \cup E_{3}$. $E$ is independent of $\left\{c_{j}\right\}$.

Next, for dilation $\lambda$ with $\lambda<-1$, we may iterate the refinement equation (1.3) 2 times to obtain a new refinement equation with the same solution, which now has $\lambda^{2}>1$ as its dilation factor. Note that $\{0,1, t\}$ remain part of the translation set for the new refinement equation. Hence $\widehat{f}_{t}$ has uniform decay for almost all $t$ except for a set of zero Hausdorff dimension.

The remaining part of this section is devoted to the proof of Proposition 3.3. For $x \in \mathbb{R}$, let $\{x\}$ denote the fractional part of $x$. 
Lemma 3.4. Let $\lambda>2$ and $x, y \in \mathbb{R}$. Suppose that $\lambda^{-n-1} \leq x-y<\lambda^{-1}$ for some $n \in \mathbb{N}$. Then there exists an integer $k$ with $0 \leq k \leq n-1$ such that $\left|\left\{\lambda^{k} x\right\}-\left\{\lambda^{k} y\right\}\right| \geq \lambda^{-2}$.

Proof. Write $x-y=a \lambda^{-k}$ for $a \in\left[\lambda^{-2}, \lambda^{-1}\right)$ and $k \in \mathbb{Z}$. From the condition $\lambda^{-(n+1)} \leq$ $x-y<\lambda^{-1}$, we obtain $0 \leq k \leq n-1$. Since $\lambda^{k} x-\lambda^{k} y=a$ with $0<a<\lambda^{-1} \leq \frac{1}{2}$, we have $\left\{\lambda^{k} x\right\}-\left\{\lambda^{k} y\right\}=a+l$ for some $l \in \mathbb{Z}$. Thus $\left|\left\{\lambda^{k} x\right\}-\left\{\lambda^{k} y\right\}\right| \geq a \geq \lambda^{-2}$.

Now for any integer $M>\lambda^{2}$ and $\mathbf{k}=\left(k_{0}, k_{1}, \ldots, k_{n-1}\right) \in Z_{M}^{n}$, where $Z_{M}:=\{0,1, \ldots, M-$ $1\}$, denote

$$
\Gamma_{T, M}(\mathbf{k})=\left\{a \in[1, T]:\left\{a \lambda^{j}\right\} \in\left[\frac{k_{j}}{M}, \frac{k_{j}+1}{M}\right) \text { for } 0 \leq j \leq n-1\right\} .
$$

It is clear that the collection $\left\{\Gamma_{T, M}(\mathbf{k}): \mathbf{k} \in Z_{M}^{n}\right\}$ is a Borel partition of the interval $[1, T]$.

Lemma 3.5. Let $\lambda>2$. For each $n \in \mathbb{N}$ and $\mathbf{k} \in Z_{M}^{n}$, the set $\Gamma_{T, M}(\mathbf{k})$ can be covered by at most $4 \lambda T+2$ intervals of length $\lambda^{-n-1}$.

Proof. By the definition of $\Gamma_{T, M}(\mathbf{k})$, for any $x, y \in \Gamma_{T, M}(\mathbf{k})$ we have

$$
\left|\left\{\lambda^{j} x\right\}-\left\{\lambda^{j} y\right\}\right| \leq \frac{1}{M}<\lambda^{-2}, \quad \forall 0 \leq j \leq n-1 .
$$

Thus by Lemma 3.4 either $|x-y| \geq \lambda^{-1}$ or $|x-y|<\lambda^{-(n+1)}$.

Now define a set $A:=\left\{x \in[1, T]: \operatorname{dist}\left(x, \Gamma_{T, M}(\mathbf{k})\right) \leq \lambda^{-n-1} / 2\right\}$. Then $A$ can be written as $\bigcup_{i} I_{i}$, where $I_{i}$ 's are disjoint intervals. Since $|x-y| \geq \lambda^{-1}$ or $|x-y|<\lambda^{-(n+1)}$ for any $x, y \in \Gamma_{T, M}(\mathbf{k})$, each interval $I_{i}$ has length not exceeding $2 \lambda^{-(n+1)}$, and the gap between any two intervals $I_{i}, I_{i^{\prime}}$ has length greater than $\frac{1}{2} \lambda^{-1}$. Thus the number of different intervals $I_{i}$ 's is less than $2 \lambda T+1$. Since each $I_{i}$ can be covered by at most two intervals of length $\lambda^{-(n+1)}$, we obtain the desired result.

Lemma 3.6. Let $\lambda>16$. There exist $\varepsilon_{0}, \delta_{0}>0$ such that for any sufficiently large $n \in \mathbb{N}$ and any $T>1$, the set $E_{\lambda}\left(n, \varepsilon_{0}, \delta_{0}\right) \cap[1, T]$ can be covered by at most $4^{n}(4 \lambda T+2)$ subintervals of $[1, T]$ of length $\lambda^{-(n+1)}$.

Proof. Pick an integer $M>\lambda^{2}$. For any $n \in \mathbb{N}$ the collection of sets $\Gamma_{T, M}(\mathbf{k})$ where $\mathbf{k}$ runs through $Z_{M}^{n}$ is a Borel partition of the interval $[1, T]$. Take $\delta_{0}=\frac{1}{M}$. Notice that $\|x\|_{\mathbb{Z}}<\delta_{0}$ if and only if $\{x\} \in\left[0, \frac{1}{M}\right)$ or $\{x\} \in\left[\frac{M-1}{M}, 1\right)$. Assume that $0<\varepsilon<1 / 4$. It follows from the definition that for $E_{\lambda}(n, \varepsilon, 1 / M) \cap \Gamma_{T, M}(\mathbf{k}) \neq \emptyset$ we must have

$$
\left|\left\{0 \leq j \leq n-1: k_{j} \notin\{0, M-1\}\right\}\right| \leq \varepsilon n
$$


Denote by $[x]$ the integral part of $x$. Let $\mathcal{B}_{n, \varepsilon}$ denote the collection of all $\mathbf{k} \in Z_{M}^{n}$ satisfying (3.3). Then an easy combinatorial argument yields

$$
\left|\mathcal{B}_{n, \varepsilon}\right|=\sum_{k=0}^{[\varepsilon n]}\left(\begin{array}{l}
n \\
k
\end{array}\right)(M-2)^{k} 2^{n-k} \leq[\varepsilon n]\left(\begin{array}{c}
n \\
{[\varepsilon n]}
\end{array}\right) M^{[\varepsilon n]} 2^{n+1} .
$$

By the Stirling formula,

$$
\left(\begin{array}{c}
n \\
{[\varepsilon n]}
\end{array}\right)=e^{n(-\varepsilon \log \varepsilon-(1-\varepsilon) \log (1-\varepsilon)+o(1))} .
$$

It follows that

$$
\left|\mathcal{B}_{n, \varepsilon}\right| \leq[\varepsilon n] M^{\varepsilon n} 2^{n+1} e^{n(-\varepsilon \log \varepsilon-(1-\varepsilon) \log (1-\varepsilon)+o(1))} .
$$

Let $\varepsilon=\varepsilon_{0}$ be small enough so that

$$
M^{\varepsilon_{0}} e^{-\varepsilon_{0} \log \varepsilon_{0}-\left(1-\varepsilon_{0}\right) \log \left(1-\varepsilon_{0}\right)}<2 .
$$

Then $\left|\mathcal{B}_{n, \varepsilon_{0}}\right| \leq 4^{n}$ for sufficiently large $n$. Since

$$
E_{\lambda}\left(n, \varepsilon_{0}, 1 / M\right) \cap[1, T] \subset \bigcup_{\mathbf{k} \in \mathcal{B}_{n, \varepsilon_{0}}} \Gamma_{T, M}(\mathbf{k}),
$$

by Lemma $3.5 E_{\lambda}\left(n, \varepsilon_{0}, 1 / M\right) \cap[1, T]$ can be covered by at most $4^{n}(4 \lambda T+2)$ subintervals of $[1, T]$ of length $\lambda^{-n-1}$. This proves the lemma.

Lemma 3.7. Let $\lambda>16$. Let $\varepsilon_{0}, \delta_{0}>0$ be as in Lemma 3.6. Then for any $T>\lambda$ the set $F_{\lambda}\left(n, \varepsilon_{0}, \delta_{0}\right) \cap\left[1, \lambda^{-1} T\right]$ can be covered by at most $4^{2 n}(4 \lambda T+2)^{2}$ subintervals of $\left[1, \lambda^{-1} T\right]$ of length $2 T \lambda^{-n-1}$.

Proof. Observe that

$$
F_{\lambda}\left(n, \varepsilon_{0}, \delta_{0}\right) \cap\left[1, \lambda^{-1} T\right] \subset\left(E_{\lambda}\left(n, \varepsilon_{0}, \delta_{0}\right) \cap[1, T]\right) /\left(E_{\lambda}\left(n, \varepsilon_{0}, \delta_{0}\right) \cap[1, \lambda]\right) .
$$

By Lemma 3.6, for any large enough $n$ the set $E_{\lambda}\left(n, \varepsilon_{0}, \delta_{0}\right) \cap[1, T]$ can be covered by at most $4^{n}(4 \lambda T+2)$ subintervals of $[1, T]$ of length $\lambda^{-(n+1)}$. Denote these intervals by $I_{i}, 1 \leq i \leq p$, where $p$ is an integer not exceeding $4^{n}(4 \lambda T+2)$. Set $B_{i, j}=\left\{x / y: x \in I_{i}, y \in I_{j}\right\}$ for $1 \leq i, j \leq p$. Then $B_{i, j}$ is an interval. It is not hard to check that each $B_{i, j}$ has length less than $2 T \lambda^{-n-1}$. Now $F_{\lambda}\left(n, \varepsilon_{0}, \delta_{0}\right) \cap\left[1, \lambda^{-1} T\right] \subset \bigcup_{1 \leq i, j \leq p} B_{i, j}$. This completes the proof of the lemma.

Proof of Proposition 3.3. By Lemma 3.7, there exist $\varepsilon_{0}, \delta_{0}>0$ such that for any fixed $T>\lambda$ and sufficiently large $n \in \mathbb{N}$, the set $F_{\lambda}\left(n, \varepsilon_{0}, \delta_{0}\right) \cap\left[1, \lambda^{-1} T\right]$ can be covered by at most $4^{2 n}(4 \lambda T+2)^{2}$ subintervals of $\left[1, \lambda^{-1} T\right]$ of length $2 T \lambda^{-n-1}$. It implies immediately that 
the Hausdorff dimension of $\bigcap_{k=1}^{\infty} \bigcup_{n=k}^{\infty} F_{\lambda}\left(n, \varepsilon_{0}, \delta_{0}\right) \cap\left[1, \lambda^{-1} T\right]$ does not exceed $\log 16 / \log \lambda$. The proposition follows since $T$ can be taken arbitrarily large.

\section{Proof of Theorem 1.6}

In this section we prove Theorem 1.6 by proving several results concerning the dilation factor $\lambda$, where $\lambda$ is an algebraic number that is the root of the irreducible polynomial $p(x)=\sum_{j=0}^{n} a_{i} x^{i}$, which has the property that all other roots of $p(x)$ are outside the unit circle. For convenience we denote $K=\left|a_{0}\right|$.

We divide the proof into two parts. In the first part we prove that $f \in L^{\infty}$. In the second part we prove $\widehat{f}$ has uniform decay at $\infty$.

To prove the first part we consider the self-similar measure $\mu$ associated with the refinement equation, which is the unique Borel probability measure satisfying the following self-similar relation:

$$
\mu=\frac{1}{K} \sum_{j=0}^{K-1} \mu \circ S_{j}^{-1},
$$

where $S_{j}(x)=\lambda^{-1}(x+j)$. It is well known that if $\mu$ is absolutely continuous then its density is precisely $f$. Thus to prove $f \in L^{\infty}$ we only need to prove that $\mu$ has a uniformly bounded density.

Lemma 4.1. Let $\mathcal{A}:=Z_{K}-Z_{K}=\{j \in \mathbb{Z}:|j|<K\}$. Then $\sum_{j=0}^{m} \varepsilon_{j} \lambda^{j}=0$ for $\varepsilon_{0}, \ldots, \varepsilon_{m} \in$ $\mathcal{A}$ if and only if $\varepsilon_{j}=0$ for all $j$.

Proof. Let $g(x)=\sum_{j=0}^{m} \varepsilon_{j} x^{j}$. Since $g(\lambda)=0$ and $p(x)=\sum_{j=0}^{n} a_{i} x^{i}$ is the minimal polynomial for $\lambda$, it follows that $p(x) \mid g(x)$. Thus $a_{0} \mid \varepsilon_{0}$. This yields $\varepsilon_{0}=0$. Factoring out $x$ in $g(x)$ and repeating the argument yield $\varepsilon_{j}=0$ for all $j$.

Lemma 4.2. Let $P(x)$ be a polynomial of degree $m$ with integer coefficients. For any $d \in \mathbb{N}$ and variables $x_{1}, x_{2}, \ldots, x_{d}$, set

$$
y_{k}=\sum_{1 \leq j_{1}<\ldots<j_{k} \leq d} x_{j_{1}} \cdots x_{j_{k}}, \quad k=1,2, \ldots, d .
$$

Then there is an integral polynomial $U\left(y_{1}, y_{2}, \ldots, y_{d}\right)$ of degree not exceeding $m$ such that

$$
\prod_{j=1}^{d} P\left(x_{j}\right)=U\left(y_{1}, y_{2}, \ldots, y_{d}\right)
$$


Proof. Note that $\prod_{j=1}^{d} P\left(x_{j}\right)$ is a symmetric polynomial with integer coefficients. It is well known (see e.g. Jacobson [10], Theorem 2.20) that it can be expressed as $U\left(y_{1}, y_{2}, \ldots, y_{d}\right)$ for some integer polynomial $U$. It remains to prove that $\operatorname{deg}(U) \leq m$. To do so we show that $\prod_{j=1}^{d} P\left(x_{j}\right)=U_{1}\left(y_{1}, y_{2}, \ldots, y_{d}\right)$ for some complex polynomial $U_{1}$ with $\operatorname{deg}\left(U_{1}\right) \leq m$. Then the uniqueness of the polynomial implies that $U=U_{1}$ (see again [10], Theorem 2.20).

Now let $P(x)=a\left(x-\alpha_{1}\right) \cdots\left(x-\alpha_{m}\right)$. Then

$$
\prod_{j=1}^{d} P\left(x_{j}\right)=a^{d} \prod_{j=1}^{d} \prod_{k=1}^{m}\left(x_{j}-\alpha_{k}\right)=a^{d} \prod_{k=1}^{m} \prod_{j=1}^{d}\left(x_{j}-\alpha_{k}\right) .
$$

It is clear that $\prod_{j=1}^{d}\left(x_{j}-\alpha_{k}\right)$ is a polynomial of $y_{1}, y_{2}, \ldots, y_{d}$ of degree 1 . Hence $\prod_{j=1}^{d} P\left(x_{j}\right)=$ $U_{1}\left(y_{1}, y_{2}, \ldots, y_{d}\right)$ for some complex polynomial of degree $\leq m$. This proves the lemma.

Lemma 4.3. There exists a constant $C>0$ such that for any $m \in \mathbb{N}$,

$$
\inf \left\{\left|\sum_{j=0}^{m} \varepsilon_{j} \lambda^{j}\right| \neq 0: \varepsilon_{j} \in \mathcal{A}\right\} \geq C|\lambda|^{m} K^{-m} .
$$

Proof. Let $\lambda_{1}=\lambda$ and $\lambda_{2}, \ldots, \lambda_{n}$ be the algebraic conjugates of $\lambda$. For any $\varepsilon_{0}, \ldots, \varepsilon_{m} \in \mathcal{A}$ set $P(x)=\sum_{j=0}^{m} \varepsilon_{j} x^{j}$. By Lemma $4.2, \prod_{j=1}^{d} P\left(x_{j}\right)$ can be written as $U\left(y_{1}, y_{2}, \ldots, y_{n}\right)$ for some integral polynomial $U$ of degree not exceeding $m$, where $y_{k}$ are given in Lemma 4.2. Now set $x_{j}=\lambda_{j}$. Then elementary algebra tells us that $y_{k} \in \frac{1}{a_{n}} \mathbb{Z}$ for all $k$, so we have $P\left(\lambda_{1}\right) P\left(\lambda_{2}\right) \ldots P\left(\lambda_{n}\right) \in \frac{1}{\left(a_{n}\right)^{m}} \mathbb{Z}$.

Now assume that $P\left(\lambda_{1}\right) \neq 0$. Then $P\left(\lambda_{k}\right) \neq 0$ for all $2 \leq k \leq n$. Thus

$$
\left|P\left(\lambda_{1}\right) P\left(\lambda_{2}\right) \ldots P\left(\lambda_{n}\right)\right| \geq \frac{1}{\left|a_{n}\right|^{m}} .
$$

Notice that for any $j$ we have

$$
\left|P\left(\lambda_{j}\right)\right| \leq(K-1)\left(1+\left|\lambda_{j}\right|+\ldots+\left|\lambda_{j}\right|^{m}\right) \leq D\left|\lambda_{j}\right|^{m}
$$

for a constant $D>0$. It follows from (4.3) and the fact $\left|\prod_{j=1}^{n} \lambda_{j}\right|=\left|a_{0} / a_{n}\right|$ that

$$
\begin{aligned}
\left|P\left(\lambda_{1}\right)\right| & \geq \frac{1}{\left|a_{n}\right|^{m} D^{n-1} \prod_{j=2}^{n}\left|\lambda_{j}\right|^{m}} \\
& =\frac{\lambda_{1}^{m}}{\left|a_{n}\right|^{m} D^{n-1} \prod_{j=1}^{n}\left|\lambda_{j}\right|^{m}} \\
& =\frac{\lambda_{1}^{m}}{D^{n-1}\left|a_{0}\right|^{m}} \\
& =C|\lambda|^{m} K^{-m} .
\end{aligned}
$$


Proposition 4.4. The self-similar measure $\mu$ is absolutely continuous with a bounded density function.

Proof. Let $\Delta$ be the support of $\mu$. It suffices to prove that there exists a constant $M>0$ such that $\mu(I) \leq M|I|$ for any subinterval $I$ of $\Delta$, where $|I|$ denotes the length of $I$. To do so we write $\mathcal{B}=\{0,1, \ldots, K-1\}$ and let $\mathcal{B}_{m}$ denote the set of all words of length $m$ over $\mathcal{B}$. For simplicity, we write $S_{\mathbf{j}}=S_{j_{0}} \circ S_{j_{1}} \ldots \circ S_{j_{m-1}}$ for $\mathbf{j}=j_{0} j_{1} \ldots j_{m-1} \in \mathcal{B}_{m}$.

Now iterating (4.1) $m$ times yields

$$
\mu(I)=\frac{1}{K^{m}} \sum_{\mathbf{j} \in \mathcal{B}_{m}} \mu \circ S_{J}^{-1}(I) .
$$

for any interval $I=[a, b] \subset \Delta$. Since $\mu$ is supported on $\Delta$, it follows that

$$
\mu(I) \leq K^{-m}\left|\left\{\mathbf{j} \in \mathcal{B}_{m}: S_{\mathbf{j}}(\Delta) \cap I \neq \emptyset\right\}\right| .
$$

Note that $S_{\mathbf{j}}(\Delta)$ has diameter $|\lambda|^{-m}|\Delta|$ where $\mid \Delta$ denotes the diameter of $\Delta$. Thus $S_{\mathbf{j}}(\Delta) \cap$ $I \neq \emptyset$ implies $S_{\mathbf{j}}(0) \in\left[a-|\lambda|^{-m}|\Delta|, b+|\lambda|^{-m}|\Delta|\right]$, where $S_{\mathbf{j}}(0)=\sum_{k=0}^{m-1} j_{k} \lambda^{-k}$. Hence by Lemma $4.3,\left|S_{\mathbf{j}}(0)-S_{\mathbf{j}^{\prime}}(0)\right| \geq C K^{-m}$ for differents indices $\mathbf{j}, \mathbf{j}^{\prime} \in \mathcal{B}_{m}$. Thus for any large integer $m$ we must have

$$
\left|\left\{\mathbf{j} \in \mathcal{B}_{m}: S_{\mathbf{j}}(\Delta) \cap I \neq \emptyset\right\}\right| \leq C^{-1} K^{m}\left(|I|+2|\lambda|^{-m}|\Delta|\right)+1 \leq 2 C^{-1} K^{m}|I| .
$$

Combining it with (4.4) yields $\mu(I) \leq 2 C^{-1}|I|$. This completes the proof of the proposition.

We now turn to the proof of the second part of Theorem 1.6, namely $\widehat{f}$ has uniform decay at infinity.

Lemma 4.5. Let $H(\xi)=\frac{1}{K} \sum_{j=0}^{K-1} e(-j \xi)$. Suppose $\xi \in \mathbb{R}$ satisfies $\|\xi\|_{\mathbb{Z}}>1 /(2 K)$ and $\|K \xi\|_{\mathbb{Z}} \leq 1 / 4$. Then $|H(\xi)| \leq 4\|K \xi\|_{\mathbb{Z}}$.

Proof. Clearly we can write $K \xi=q+\|K \xi\|_{\mathbb{Z}}$ for some $q \in \mathbb{Z}$, and since $\|\xi\|_{\mathbb{Z}}>1 /(2 K)$ we have $\xi=p+\frac{j}{K}+\frac{1}{K}\|K \xi\|_{\mathbb{Z}}$ for some $p \in \mathbb{Z}$ and $j \in\{1, \ldots, K-1\}$. Notice that $|H(\xi)|=\frac{|\sin (K \pi \xi)|}{K|\sin (\pi \xi)|}$. Thus

$$
|H(\xi)|=\frac{\left|\sin \left(\pi\|K \xi\|_{\mathbb{Z}}\right)\right|}{K\left|\sin \left(\pi\|\xi\|_{\mathbb{Z}}\right)\right|} .
$$


The lemma is proved from the inequalities $\left|\sin \left(\pi\|K \xi\|_{\mathbb{Z}}\right)\right| \leq \pi\|K \xi\|_{\mathbb{Z}}$ and $K\left|\sin \left(\pi\|\xi\|_{\mathbb{Z}}\right)\right| \geq$ $K \pi \frac{1}{4 K}=\frac{\pi}{4}$.

Lemma 4.6. There exist $\ell \in \mathbb{N}$ and integers $b_{0}, b_{1}, \ldots, b_{\ell}$ with $\left|b_{0}\right|>\sum_{j=1}^{\ell}\left|b_{j}\right|$, such that $\lambda$ is a root of the polynomial $\sum_{j=0}^{\ell} b_{j} x^{j}$.

Proof. The lemma is obviously true when the degree of $p(x)$, the minimal polynomial of $\lambda$, is equal to one. So we assume that the degree of $\lambda$ is larger than 1 .

Denote $\lambda_{1}=\lambda$ and let $\lambda_{2}, \ldots, \lambda_{n}$ be the algebraic conjugates of $\lambda$. Then for any $m \in \mathbb{N}$, $\lambda_{j}^{m}(j=1, \ldots, n)$ are roots of an integral polynomial $P_{m}(x)=\sum_{j=0}^{n} a_{j, m} x^{j}$. Since $\left|\lambda_{j}\right|>1$ for all $j$, for sufficiently large $k$ we have $\left|\lambda_{j}\right|^{k}>2^{n}$ for all $j$. For such a $k$ we have

$$
\left|\prod_{j=1}^{n} \lambda_{j}^{k}\right|>\sum_{u=1}^{n-1}\left|\sum_{1 \leq j_{1}<\ldots<j_{u} \leq n} \lambda_{j_{1}}^{k} \cdots \lambda_{j_{u}}^{k}\right| .
$$

The above inequality implies $\left|a_{0, k}\right|>\sum_{j=1}^{n}\left|a_{j, k}\right|$. Since $\lambda$ is a root of the polynomial $P_{k}\left(x^{k}\right)=\sum_{j=0}^{n} a_{j, k} x^{k j}$, we obtain the desired result.

The following proposition is the key to complete the proof of our theorem.

Proposition 4.7. There exist $0<\varepsilon<1 /(4 K), 0<\rho<1$ and $N \in \mathbb{N}$ such that for any $\xi \in \mathbb{R}$ with $|\xi|>|\lambda|^{N}$, if $\left\|\lambda^{-j} \xi\right\|_{\mathbb{Z}}<\varepsilon$ for $j=1, \ldots, N$, then there exists an integer $m>N$ such that

$$
\left\|\lambda^{-m} \xi\right\|_{\mathbb{Z}}>\frac{1}{2 K} \quad \text { and } \quad\left\|K \lambda^{-m} \xi\right\|_{\mathbb{Z}} \leq \frac{\rho^{m}}{4}
$$

Proof. Recall that $\lambda$ is a root of the irreducible integral polynomial $\sum_{j=0}^{n} a_{j} x^{j}$. By Lemma 4.6 , it is also a root of an integral polynomial $\sum_{j=0}^{\ell} b_{j} x^{j}$ with $\left|b_{0}\right| \geq \sum_{j=1}^{\ell}\left|b_{j}\right|$. Without loss of generality we assume that $a_{0}>0$ and $b_{0}>0$. Clearly,

$$
-1=\frac{1}{a_{0}} \sum_{j=1}^{n} a_{j} \lambda^{j} \quad \text { and } \quad-1=\frac{1}{b_{0}} \sum_{j=1}^{\ell} b_{j} \lambda^{j} .
$$

Now for any $x \in \mathbb{R}$ let $\llbracket x \rrbracket$ denote the integer that is closest to $x$ (especially let $\llbracket n+1 / 2 \rrbracket=n$ for $n \in \mathbb{Z})$, and let $\rrbracket x \llbracket=x-\llbracket x \rrbracket$. Clearly we have $|\rrbracket x \llbracket|=\|x\|_{\mathbb{Z}}$. Thus for any $x \in \mathbb{R}$ and $k \in \mathbb{N}$, we have

$$
-\lambda^{-k} x=\frac{1}{a_{0}} \sum_{j=1}^{n} a_{j} \lambda^{-k+j} x=\frac{1}{a_{0}} \sum_{j=1}^{n} a_{j} \llbracket \lambda^{-k+j} x \rrbracket+\frac{1}{a_{0}} \sum_{j=1}^{n} a_{j} \rrbracket \lambda^{-k+j} x \llbracket
$$


and

$$
-\lambda^{-k} x=\frac{1}{b_{0}} \sum_{j=1}^{\ell} b_{j} \lambda^{-k+j} x=\frac{1}{b_{0}} \sum_{j=1}^{\ell} b_{j} \llbracket \lambda^{-k+j} x \rrbracket+\frac{1}{b_{0}} \sum_{j=1}^{\ell} b_{j} \rrbracket \lambda^{-k+j} x \llbracket .
$$

For convenience, we write

$$
A_{k}(x)=\frac{1}{a_{0}} \sum_{j=1}^{n} a_{j} \llbracket \lambda^{-k+j} x \rrbracket, \quad B_{k}(x)=\frac{1}{a_{0}} \sum_{j=1}^{n} a_{j} \rrbracket \lambda^{-k+j} x \llbracket
$$

and

$$
C_{k}(x)=\frac{1}{b_{0}} \sum_{j=1}^{\ell} b_{j} \llbracket \lambda^{-k+j} x \rrbracket, \quad D_{k}(x)=\frac{1}{b_{0}} \sum_{j=1}^{\ell} b_{j} \rrbracket \lambda^{-k+j} x \llbracket .
$$

It is clear that $A_{k}(x) \in \frac{1}{a_{0}} \mathbb{Z}, C_{k}(x) \in \frac{1}{b_{0}} \mathbb{Z}$ and (4.6), (4.7) can be rewritten as

$$
-\lambda^{-k} x=A_{k}(x)+B_{k}(x)=C_{k}(x)+D_{k}(x) .
$$

Denote $\eta_{1}=\frac{1}{a_{0}} \sum_{j=1}^{n}\left|a_{i}\right|$ and $\eta_{2}=\frac{1}{b_{0}} \sum_{j=1}^{\ell}\left|b_{j}\right|$. Then $0<\eta_{2}<1$. We shall choose an $\varepsilon>0$ that is sufficiently small.

Now fix $\xi \in \mathbb{R}$ so that $|\xi| \geq|\lambda|^{\ell}$ and $\left\|\lambda^{-j} \xi\right\|_{\mathbb{Z}}<\varepsilon$ for all $1 \leq j \leq \ell$. In the following we prove that there exist a $\rho \in(0,1)$ (independent of $\xi$ ) and an integer $m>\ell$ such that $\left\|\lambda^{-m} \xi\right\|_{\mathbb{Z}}>\frac{1}{2 a_{0}}$ and $\left\|a_{0} \lambda^{-m} \xi\right\|_{\mathbb{Z}}<\rho^{m} / 4$.

We first claim that there exists an integer $m>\ell$ such that $C_{m}(x) \notin \mathbb{Z}$. Assume on the contrary that the claim is not true. Then $C_{k}(\xi) \in \mathbb{Z}$ for any integer $k>\ell$. By (4.8), for all $k \geq \ell+1$ we have

$$
\begin{aligned}
\left\|\lambda^{-k} \xi\right\|_{\mathbb{Z}} & \leq\left|D_{k}(\xi)\right| \leq \frac{1}{b_{0}} \sum_{j=1}^{\ell}\left|b_{j}\right|\left\|\lambda^{-k+j} \xi\right\|_{\mathbb{Z}} \\
& \leq \eta_{2} \max \left\{\left\|\lambda^{-k+j} \xi\right\|_{\mathbb{Z}}: 1 \leq j \leq \ell\right\} .
\end{aligned}
$$

Since $\left\|\lambda^{-j} \xi\right\|_{\mathbb{Z}}<\varepsilon$ for $1 \leq j \leq \ell$, by (4.9) and an inductive argument we have $\left\|\lambda^{-k} \xi\right\|_{\mathbb{Z}}<\varepsilon$ for all $k \geq \ell+1$. However since $|\xi|>|\lambda|^{\ell}$, there exists some integer $k_{0}>\ell$ such that $\left|\lambda^{-k_{0}} \xi\right| \in$ $\left[|\lambda|^{-2},|\lambda|^{-1}\right)$. Therefore with $\varepsilon$ small we have $\left\|\lambda^{-k_{0}} \xi\right\|_{\mathbb{Z}} \geq \min \left\{\left\|\lambda^{-2}\right\|_{\mathbb{Z}},\left\|\lambda^{-1}\right\|_{\mathbb{Z}}\right\}>\varepsilon$, which leads to a contradiction. This finishes the claim.

Now assume without loss of generality that $m$ is the smallest integer so that $m>\ell$ and $C_{m}(\xi) \notin \mathbb{Z}$. We consider the following two cases separately.

Case 1. $m=\ell+1$. 
In this case we have

$$
\left|D_{\ell+1}(\xi)\right| \leq \eta_{2} \max \left\{\left\|\lambda^{-(\ell+1-j)}\right\|_{\mathbb{Z}}: 1 \leq j \leq \ell\right\} \leq \eta_{2} \varepsilon
$$

and with $\varepsilon$ small enough,

$$
\left\|\lambda^{-\ell-1} \xi\right\|_{\mathbb{Z}} \geq\left\|C_{\ell+1}(\xi)\right\|_{\mathbb{Z}}-\left\|D_{\ell+1}(\xi)\right\|_{\mathbb{Z}} \geq \frac{1}{b_{0}}-\eta_{2} \varepsilon \geq \frac{1}{2 b_{0}} .
$$

Case 2. $m \geq \ell+2$.

Write $m=2+p \ell+q$, where $p \in \mathbb{N}$ and $0 \leq q \leq \ell-1$. Since $C_{k}(\xi) \in \mathbb{Z}$ for $\ell+1 \leq k \leq m-1$, as with (4.9) we have

$$
\left\|\lambda^{-k} \xi\right\|_{\mathbb{Z}} \leq \eta_{2} \max \left\{\left\|\lambda^{-k+j} \xi\right\|_{\mathbb{Z}}: 1 \leq j \leq \ell\right\} \quad \text { for all } \ell+1 \leq k \leq m-1 .
$$

Using (4.10) and induction we have

$$
\left\|\lambda^{-t \ell-j} \xi\right\|_{\mathbb{Z}} \leq \eta_{2}^{t} \varepsilon
$$

for any integers $t, j$ such that $1 \leq t \leq p, 1 \leq j \leq \ell$ and $t \ell+j \leq m-1$. Particularly

$$
\left\|\lambda^{-m+j} \xi\right\|_{\mathbb{Z}} \leq \eta_{2}^{p-1} \varepsilon, \quad j=1,2, \ldots, \ell .
$$

Thus

$$
\left|D_{m}(\xi)\right| \leq \eta_{2} \max \left\{\left\|\lambda^{-(m-j)} \xi\right\|_{\mathbb{Z}}: 1 \leq j \leq \ell\right\} \leq \eta_{2}^{p} \varepsilon \leq \eta_{2}^{\frac{m}{2 \ell}} \varepsilon
$$

and with $\varepsilon$ small enough,

$$
\left\|\lambda^{-m} \xi\right\|_{\mathbb{Z}} \geq\left\|C_{m}(\xi)\right\|_{\mathbb{Z}}-\left\|D_{m}(\xi)\right\|_{\mathbb{Z}} \geq \frac{1}{b_{0}}-\varepsilon \geq \frac{1}{2 b_{0}}
$$

Take $\rho=\eta_{2}^{\frac{1}{2 \ell}}$. We have proved that in each case there always exists an integer $m \geq \ell+1$ such that $\left\|\lambda^{-m} \xi\right\|_{\mathbb{Z}} \geq \frac{1}{2 b_{0}},\left\|b_{0} \lambda^{-m} \xi\right\|_{\mathbb{Z}} \leq b_{0} \rho^{m} \varepsilon$ and $\left\|\lambda^{-m+j} \xi\right\|_{\mathbb{Z}}<\varepsilon$ for all $1 \leq j \leq \ell$. Observe that

$$
\left|B_{m}(\xi)\right| \leq \eta_{1} \max \left\{\left\|\lambda^{-(m-j)} \xi\right\|_{\mathbb{Z}}: \quad 1 \leq j \leq \ell\right\} \leq \eta_{1} \varepsilon
$$

From the fact $A_{m}(\xi)+B_{m}(\xi)=C_{m}(\xi)+D_{m}(\xi)$ we have

$$
\left|A_{m}(\xi)-C_{m}(\xi)\right|=\left|B_{m}(\xi)-D_{m}(\xi)\right| \leq\left(\eta_{1}+1\right) \varepsilon<\frac{1}{a_{0} b_{0}} .
$$

It implies that $A_{m}(\xi)-C_{m}(\xi)=0$ since $A_{m}(\xi) \in \frac{1}{a_{0}} \mathbb{Z}$ and $C_{m}(\xi) \in \frac{1}{b_{0}} \mathbb{Z}$. Hence $B_{m}(\xi)=$ $D_{m}(\xi)$. By making $\varepsilon$ small it follows that

$$
\left\|a_{0} \lambda^{-m} \xi\right\|_{\mathbb{Z}} \leq\left|a_{0} B_{m}(\xi)\right|=\left|a_{0} D_{m}(\xi)\right| \leq a_{0} \rho^{m} \varepsilon \leq \frac{\rho^{m}}{4} .
$$


Since $C_{m}(\xi) \notin \mathbb{Z}$, so does $A_{m}(\xi)$. Hence $\left\|A_{m}(\xi)\right\|_{\mathbb{Z}} \geq \frac{1}{a_{0}}$

$$
\left\|\lambda^{-m} \xi\right\|_{\mathbb{Z}} \geq\left\|A_{m}(\xi)\right\|_{\mathbb{Z}}-\left\|B_{m}(\xi)\right\|_{\mathbb{Z}} \geq \frac{1}{a_{0}}-\eta_{1} \varepsilon>\frac{1}{2 a_{0}} .
$$

This finishes the proof of the proposition.

Proof of Theorem 1.6. Let $f$ be the unique compactly supported distribution of (1.4) with $\hat{f}(0)=1$. Let $\varepsilon, \rho$ and $N$ be given in Proposition 4.7. To prove that $\hat{f}$ has a uniform decay at infinity, we only need to show that there exists a $\delta>0$ such that for any $\xi \in \mathbb{R}$ with $|\xi|>|\lambda|^{N}$, there exists an $\ell \in \mathbb{N}$ such that

$$
|\hat{f}(\xi)| \leq \lambda^{-\ell \delta}\left|\hat{f}\left(\lambda^{-\ell} \xi\right)\right|
$$

Now $\hat{f}(\xi)=\hat{f}(\xi / \lambda) H(\xi / \lambda)$, where $H(\xi)$ is defined as in Lemma 4.5. For any $k \in \mathbb{N}$, iterating the above equality $k$ times yields $\hat{f}(\xi)=\hat{f}\left(\lambda^{-k} \xi\right) H\left(\lambda^{-1} \xi\right) \ldots H\left(\lambda^{-k} \xi\right)$. Using the inequality $|H(x)| \leq 1$ we have

$$
|\hat{f}(\xi)| \leq\left|\hat{f}\left(\lambda^{-k} \xi\right)\right|\left|H\left(\lambda^{-k} \xi\right)\right|, \quad \forall \xi \in \mathbb{R}, k \in \mathbb{N} .
$$

Now set $C:=\max \left\{|H(\xi)|:\|\xi\|_{\mathbb{Z}} \geq \varepsilon\right\}$. It is clear $0<C<1$. According to Proposition 4.7, for any $\xi \in \mathbb{R}$ with $|\xi|>|\lambda|^{N}$, either $\left\|\lambda^{-j} \xi\right\|_{\mathbb{Z}} \geq \varepsilon$ for some $1 \leq j \leq N$, or there exists $m>N$ such that $\left\|\lambda^{-m} \xi\right\|_{\mathbb{Z}}>\frac{1}{2 K}$ and $\left\|K \lambda^{-m} \xi\right\|_{\mathbb{Z}}<\rho^{m} / 4$. By Lemma 4.5, either $\left|H\left(\lambda^{-j} \xi\right)\right| \leq C$ for some $1 \leq j \leq N$, or $\left|H\left(\lambda^{-m} \xi\right)\right| \leq 4\left\|K \lambda^{-m} \xi\right\|_{\mathbb{Z}} \leq \rho^{m}$ for some $m>N$. Define

$$
\delta=\min \left\{\frac{\log (1 / C)}{N \log \lambda}, \frac{\log (1 / \rho)}{\log \lambda}\right\} .
$$

Then for any $\xi \in \mathbb{R}$ with $|\xi|>\lambda^{N}$, there exist $\ell \in \mathbb{N}$ such that $H\left(\lambda^{-\ell} \xi\right) \leq \lambda^{-\ell \delta}$. The theorem now follows from (4.11).

\section{Appendix: Open Questions and Results of Erdös and Kahane}

In this section we prove that compactly supported refinable distributions with integer translations do not have uniform decay at infinity if the dilations are Pisot or Salem numbers. This result was established in the case of Bernoulli convolutions by Erdös ([6], Pisot numbers) and Kahane ([11], Salem numbers). The general case stated here is proved using Kahane's technique, although some nontrivial technical details had to be overcome. 
Proposition 5.1. Let $f(x)$ be the refinable distribution given by

$$
f(x)=\sum_{j=0}^{n} c_{j} f\left(\lambda x-d_{j}\right), \quad \sum_{j=0}^{n} c_{j}=|\lambda|
$$

where $\lambda$ is a Pisot number or Salem number with $|\lambda|>1$ and $\lambda \notin \mathbb{Z}, c_{j} \neq 0$ and $d_{j} \in \mathbb{Z}$ for all $j$. Then $\hat{f}$ does not have uniform decay at infinity.

To prove this proposition, we need the following result, which was first proved by Pisot (the reader may see [15] for a proof).

Theorem 5.2 (Pisot [14]). Let $\lambda$ be an arbitrary algebraic integer. Then there exists a Pisot number in $\mathbb{Z}[\lambda]$ having the same degree as $\lambda$.

Corollary 5.3. Let $\lambda$ be a Pisot or Salem number. Then there exists a sequence $\left\{u_{m}\right\}$ in $\mathbb{Z}[\lambda]$ such that $\left\|\lambda^{k} u_{m}\right\|_{\mathbb{Z}}<\frac{1}{m}$ for all $m, k \in \mathbb{N}$. Furthermore, if $\lambda$ is a Pisot number then we may take $u_{m}=m \lambda^{\alpha_{m}}$ for some $\alpha_{m} \in \mathbb{N}$. If $\lambda$ is a Salem number then we may take $u_{m}=\omega^{\alpha_{m}}$ for some $\alpha_{m} \in \mathbb{N}$, where $\omega \in \mathbb{Z}[\lambda]$ is a Pisot number independent of $m$.

Proof. Assume that $\lambda$ is of degree $d$. Let $\lambda_{1}, \ldots, \lambda_{d-1}$ be the algebraic conjugates of $\lambda$. Observe that if $f(x) \in \mathbb{Z}[x]$ then $f(\lambda)+\sum_{j=1}^{d-1} f\left(\lambda_{j}\right) \in \mathbb{Z}$. In particular $\|f(\lambda)\|_{\mathbb{Z}} \leq$ $\sum_{j=1}^{d-1}\left|f\left(\lambda_{j}\right)\right|$.

If $\lambda$ is a Pisot number. Then $\left|\lambda_{j}\right|<1$. Choose $\alpha_{m}>0$ so that $\sum_{j=1}^{d-1} m\left|\lambda_{j}\right|^{\alpha_{m}}<\frac{1}{m}$. Then

$$
\left\|\lambda^{k} u_{m}\right\|_{\mathbb{Z}}=\left\|m \lambda^{k+\alpha_{m}}\right\|_{\mathbb{Z}} \leq \sum_{j=1}^{d-1} m\left|\lambda_{j}\right|^{\alpha_{m}+k} \leq \frac{1}{m} .
$$

If $\lambda$ is a Salem number. Let $\omega=f(\lambda)$ be a Pisot number of the same degree as $\lambda$, where $f(x) \in \mathbb{Z}[x]$. Then the algebraic conjugates of $\omega$ are $\omega_{j}:=f\left(\lambda_{j}\right)$. Let $\alpha_{m}>0$ such that $\sum_{j=1}^{d-1}\left|f\left(\lambda_{j}\right)\right|^{\alpha_{m}}<\frac{1}{m}$. It follows from the property that $\left|\lambda_{j}\right| \leq 1$ that

$$
\left\|\lambda^{k} u_{m}\right\|_{\mathbb{Z}}=\left\|\lambda^{k} \omega^{\alpha_{m}}\right\|_{\mathbb{Z}} \leq \sum_{j=1}^{d-1}\left|\lambda_{j}\right|^{k}\left|f\left(\lambda_{j}\right)\right|^{\alpha_{m}} \leq \frac{1}{m} .
$$

Proof of Proposition 5.1. Assume that Proposition 5.1 is not true. Then $\hat{f}$ has a $\gamma$ uniform decay for some $\gamma>0$. Let $P(\xi)$ be the symbol of the refinement equation (5.1). Since $P(n)=1$ for any integer $n$, we may choose $m_{0}$ sufficiently large so that $\left|P\left(\lambda^{k} u_{m}\right)\right|>$ 
$|\lambda|^{-\gamma / 2}$ for all $m>m_{0}$ and $k \geq 0$, where $u_{m}$ is as in Corollary 5.3. We claim that $\hat{f}\left(u_{m}\right)=0$ for all $m>m_{0}$. Assume it is false, i.e. $\hat{f}\left(u_{m}\right) \neq 0$ for some $m>m_{0}$. Then for any $N \in \mathbb{N}$,

$$
\left|\hat{f}\left(\lambda^{N} u_{m}\right)\right|=\left|\hat{f}\left(u_{m}\right)\right| \prod_{j=0}^{N-1}\left|P\left(\lambda^{j} u_{m}\right)\right| \geq|\lambda|^{-\gamma N / 2} \hat{f}\left(u_{m}\right) .
$$

However the above inequality contradicts the fact that $\hat{f}$ has a $\gamma$-uniform decay. This proves the claim.

Now $\hat{f}\left(u_{m}\right)=0$ implies that there exists a $j_{m} \in \mathbb{N}$ such that $P\left(u_{m} \lambda^{-j_{m}}\right)=0$. We now consider the case that $\lambda$ is a Pisot number. In this case $u_{m}=m \lambda^{\alpha_{m}}$. Set $k_{m}=j_{m}-\alpha_{m}$. Then $P\left(m \lambda^{-k_{m}}\right)=0$ for all $m>m_{0}$. Let $K$ be an integer such that $\lambda^{-1} \in \frac{1}{K} \mathbb{Z}[\lambda]$. Then $\lambda^{-k_{m}} \in K^{-k_{m}} \mathbb{Z}[\lambda]$. Write

$$
\lambda^{-k_{m}}=K^{-k_{m}}\left(p_{m, 0}+p_{m, 1} \lambda+\cdots+p_{m, d-1} \lambda^{d-1}\right),
$$

where $d$ is the degree of $\lambda$ and $p_{m, i} \in \mathbb{Z}$ for all $i$. This expression is unique since $\left\{\lambda^{i}: 0 \leq\right.$ $i<d\}$ are linearly independent over $\mathbb{Q}$. Hence

$$
m \lambda^{-k_{m}}=K^{-k_{m}}\left(m p_{m, 0}+m p_{m, 1} \lambda+\cdots+m p_{m, d-1} \lambda^{d-1}\right) .
$$

Since $\lambda^{-k_{m}} \notin \mathbb{Q}$, at least one of $p_{m, i} \neq 0$ for some $1 \leq i \leq d-1$. Now $P(\xi)$ is a trigonometric polynomial, so $\left\{m \lambda^{-k_{m}}(\bmod 1): m>m_{0}\right\}$ is a finite set. Thus again by the linear independence of $\left\{\lambda^{i}: 0 \leq i<d\right\}$ over $\mathbb{Q}$ we know that the set

$$
\left\{\left(K^{-k_{m}} m p_{m, 1}, \ldots, K^{-k_{m}} m p_{m, d-1}\right): m>m_{0}\right\}
$$

is a finite set. But this is not true, because we may take $m$ sufficiently large and coprime with $K$ so that the nonzero numerators in $\left(K^{-k_{m}} m p_{m, 1}, \ldots, K^{-k_{m}} m p_{m, d-1}\right)$ become arbitrarily large. This yields a contradiction.

Next we consider the case that $\lambda$ is a Salem number. In this case, $u_{m}=\omega^{\alpha_{m}}$ where $\omega=f(\lambda) \in \mathbb{Z}[\lambda]$ is a Pisot number. Hence for each $m>m_{0}$ we have $P\left(\lambda^{-j_{m}} \omega^{\alpha_{m}}\right)=0$ for some $j_{m}>0$. Again, $\left\{\lambda^{-j_{m}} \omega^{\alpha_{m}}(\bmod 1): m>m_{0}\right\}$ is a finite set. Choose $m, n$ such that $\alpha_{m} \neq \alpha_{n}$ are sufficiently large and

$$
\lambda^{-j_{m}} \omega^{\alpha_{m}}-\lambda^{-j_{n}} \omega^{\alpha_{n}}=\ell \in \mathbb{Z} .
$$

Without loss of generality assume that $j_{m} \geq j_{n}$. Then $\omega^{\alpha_{m}}-\lambda^{j_{m}-j_{n}} \omega^{\alpha_{n}}=\ell \lambda^{j_{m}}$. However, this is a contradiction because for sufficiently large $\alpha_{m}, \alpha_{n}$ the left hand side is a Pisot number but the right hand side isn't. This completes the proof of the proposition. 
There are a number of interesting open questions. We list some of them here.

(1) Is it true that uniform decay in $\widehat{f}$ (assuming integer translations) can always be achieved for dilations that are not Pisot or Salem numbers? A related questions is whether Pisot numbers are the only ones that give singular Bernoulli convolutions. This question is known to be hard.

(2) Can one find another family of dilations for which refinable functions with uniform decay property in $\widehat{f}$ can be constructed explicitly?

(3) Fix the translations and weights (nonnegative) of a refinement equation, is it true that by varying the dilation $\lambda$ the resulting refinable distribution has uniform decay in $\widehat{f}$ for almost all $\lambda>1$ ?

\section{REFERENCES}

[1] A. S. Cavaretta, W. Dahmen and C. A. Micchelli, Stationary subdivision. Mem. Amer. Math. Soc. 93 (1991), no. 453.

[2] J. B. Conway, Functions of one complex variable. Second edition. Graduate Texts in Mathematics, 11. Springer-Verlag, New York-Berlin, 1978.

[3] I. Daubechies, Orthonormal bases of compactly supported wavelets, Comm. Pure and Appl. Math. 41 (7) (1988), 909-996.

[4] I. Daubechies, Ten lectures on wavelets. CBMS-NSF Regional Conference Series in Applied Mathematics, 61. Society for Industrial and Applied Mathematics (SIAM), Philadelphia, PA, 1992.

[5] P. Erdös, On a family of symmetric Bernoulli convolutions. Amer. J. Math. 61 (1939), 974-976.

[6] P. Erdös, On the smoothness properties of a family of Bernoulli convolutions. Amer. J. Math. 62 (1940), $180-186$.

[7] D.-J. Feng and Y. Wang, Bernoulli convolutions associated with certain non-Pisot numbers, Adv. Math. 187 (2004), 173-194.

[8] K. J. Falconer, Fractal Geometry. Mathematical Foundations and Applications. John Wiley \& Sons, Ltd., Chichester, 1990.

[9] A. M. Garsia, Arithmetic properties of Bernoulli convolutions. Trans. Amer. Math. Soc. 102 (1962), 409-432.

[10] N. Jacobson, Basic Algebra I, W. H. Freeman and Co, 1974.

[11] J.-P. Kahane, Sur la distribution de certaines séries aléatoires. (French) Colloque de Théorie des Nombres (Univ. Bordeaux, Bordeaux, 1969), Bull. Soc. Math. France, Mémoire. 25 (1971), 119-122.

[12] J. C. Lagarias and Y. Wang, Self-affine tiles in $\mathbb{R}^{n}$. Adv. Math. 121 (1996), 21-49.

[13] W. Lawton, S. L. Lee and Z. Shen, Characterization of compactly supported refinable splines. Adv. Comput. Math. 3 (1995), 137-145.

[14] C. Pisot, La répartition modulo 1 et les nombres algébriques, Ann. Di. Sc. Norm. Sup. Piso, Ser 2, 7(1938), 205-248.

[15] R. Salem, Algebraic numbers and Fourier Analysis. D. C. Heath and Company, 1963.

[16] Y. Peres and W. Schlag, Smoothness of projections, Bernoulli convolutions, and the dimension of exceptions. Duke Math. J. 102 (2000), 193-251.

[17] Y. Peres, W. Schlag and B. Solomyak, Sixty years of Bernoulli convolutions. Fractal geometry and stochastics, II (Greifswald/Koserow, 1998), 39-65, Progr. Probab., 46, Birkhäuser, Basel, 2000.

[18] W. Rudin, Real and complex analysis. Third edition. McGraw-Hill Book Co., New York, 1987.

[19] B. Solomyak, On the random series $\sum \pm \lambda^{n}$ (an Erdös problem). Ann. of Math. (2) 142 (1995), 611-625. 
Department of Applied Mathematics, Zhejiang University of Technology, Hangzhou, 310014 , P. R. China.

E-mail address: Daixinrong@zjut.edu.cn

Department of Mathematics, The Chinese University of Hong Kong, Shatin, Hong Kong and Department of Mathematical Sciences, Tsinghua University, Beijing 100084, People's Republic OF CHINA

E-mail address: djfeng@math.cuhk.edu.hk, dfeng@math.tsinghua.edu.cn

School of Mathematics, Georgia Institute of Technology, Atlanta, Georgia 30332, USA.

E-mail address: wang@math.gatech.edu 